\title{
On Selective Unboundedness of VASS
}

\author{
Stéphane Demri \\ LSV, CNRS, ENS de Cachan, INRIA, France
}

\begin{abstract}
Numerous properties of vector addition systems with states amount to checking the (un)boundedness of some selective feature (e.g., number of reversals, run length). Some of these features can be checked in exponential space by using Rackoff's proof or its variants, combined with Savitch's theorem. However, the question is still open for many others, e.g., reversal-boundedness. In the paper, we introduce the class of generalized unboundedness properties that can be verified in exponential space by extending Rackoff's technique, sometimes in an unorthodox way. We obtain new optimal upper bounds, for example for place boundedness problem, reversal-boundedness detection (several variants exist), strong promptness detection problem and regularity detection. Our analysis is sufficiently refined so as we also obtain a polynomial-space bound when the dimension is fixed.
\end{abstract}

\section{Introduction}

Reversal-boundedness. A standard approach to circumvent the undecidability of the reachability problem for counter automata [19] consists in designing subclasses with simpler decision problems. For instance, the reachability problem is decidable for vector addition systems with states (VASS) [15] or for lossy counter automata [1]. Among the other interesting subclasses of counter automata, reversalbounded counter automata verify that any counter has a bounded number of reversals, alternations between a nonincreasing mode and a nondecreasing mode, and vice versa. Reversal-boundedness remains a standard concept that was initially introduced in [3] for multistack automata. A major property of such operational models is that reachability sets are effectively definable in Presburger arithmetic [13], which allows decision procedures for LTL existential model-checking and other related problems, see e.g. [5]. However, many natural problems related to verification remain undecidable for reversal-bounded counter automata, see e.g. [5, 6], and the class of reversal-bounded counter automata is not recursive [13]. A significant breakthrough was achieved in [8] by designing a procedure to determine when a VASS is reversal-bounded (or weakly reversal-bounded as defined later), even though the decision procedure can be nonprimitive recursive in the worst-case. This means that reversal-bounded VASS can benefit from the known techniques for Presburger arithmetic in order to solve their verification problems.

Selective unboundedness. In order to characterize the complexity of detecting reversal-boundedness on VASS (the initial motivation for this work), we make a detour to selective unboundedness, as explained below. Numerous properties of vector addition systems with states amounts to checking the (un)boundedness of some selective feature. Some of these features can be verified in exponential space by using Rackoff's proof or its variants, whereas the question is still open for many of them. In the paper, we advocate that many properties can be decided as soon as we are able to decide selective unboundedness, which is a generalization of place unboundedness for Petri nets (known to be equivalent to VASS). The boundedness problem was first considered in [15] and shown decidable by simply inspecting Karp and Miller trees: the presence of the infinity value $\infty$ (also denoted by $\omega$ ) is equivalent to unboundedness. So, unboundedness is equivalent to the existence of a witness run of the form $\overrightarrow{x_{0}} \stackrel{*}{\rightarrow} \overrightarrow{x_{1}} \stackrel{\pi}{\rightarrow} \overrightarrow{x_{2}}$ such that $\overrightarrow{x_{1}} \prec \overrightarrow{x_{2}}$ ( $\prec$ is the standard strict ordering on tuples of natural numbers). In [21], it is shown that if there is such a run, there is one of length at most doubly exponential. This leads to the EXPSPACE-completeness

Y.-F. Chen and A. Rezine (Eds.): The 12th INFINITY Workshop. EPTCS 39, 2010, pp. 1-15 doi 10.4204/EPTCS.39.1 (c) S. Demri

This work is licensed under the Creative Commons Attribution License. 
On Selective Unboundedness of VASS

of the boundedness problem for VASS using the lower bound from [17] and Savitch's theorem. A variant problem consists in checking whether the $i$ th component is bounded, i.e., is there a bound $B$ such that for every configuration reachable from $\vec{x}_{0}$, its $i$ th component is bounded by $B$ ? Again, inspecting Karp and Miller trees reveals the answer: the presence of the infinity value $\infty$ at the $i$ th position of some extended configuration is equivalent to $i$-unboundedness. Surprisingly, the literature often mentions this alternative problem, see e.g. [22], but never specifies its complexity: EXPSPACE-hardness can be obtained from [17] but as far as we know, no elementary complexity upper bound has been shown. It might be explained by the fact that, if a VASS is unbounded, then there is a witness infinite run with an infinite number of distinct configurations. By contrast, it may happen that a VASS is $i$-unbounded but no infinite run has an infinite amount of distinct values at the $i$ th position of the configurations of the run. In the paper, we present a generalization of place unboundedness by checking whether a set of components is simultaneously unbounded, possibly with some ordering (see Section 3.2). This amounts to specifying in the Karp and Miller trees, the ordering with which the value $\infty$ appears in the different components.

Our contribution. In the paper we show the following results.

1. Detecting whether a VASS is reversal-boundedness in the sense of [13] or [8] is EXPSPACEcomplete by refining the decidability results from [8] (see Theorem 5.2).

2. We introduce the generalized unboundedness problem in which many problems can be captured such as the reversal-boundedness detection problems, the place boundedness problem, termination, strong promptness detection problem, regularity detection and many other decision problems on VASS. We show that this problem can be solved in exponential space by adapting [21] even though it does not fall into the class of increasing path formulae introduced in [2] (see Theorem 4.5).

3. Consequently, we show that regularity and strong promptness detection problems for VASS are in EXPSPACE. The EXPSPACE upper bound has been left open in [2]. Even though most of our results essentially rest on the fact that place boundedness can be solved in EXPSPACE, our slight generalization is introduced to obtain new complexity upper bound for other related problems.

4. As a by-product of our analysis and following a parameterized analysis initiated in [24, 12], for all the above-mentionned problems, we show that fixing the dimension of the VASS allows to get a PSPACE upper bound.

The paper has also original contributions as far as proof techniques are concerned. First, simultaneous unboundedness has a simple characterization in terms of Karp and Miller trees, but we provide in the paper a witness run characterization, which allows us to provide a complexity analysis along the lines of [21]. We also provide a witness pseudo-run characterization in which we sometimes admit negative component values. This happens to be the right approach when a characterization from coverability graphs [15, 28] already exists. Apart from this unorthodox adaptation of [21], in the counterpart of Rackoff's proof about the induction on the dimension, we provide an induction on the dimension and on the length of the properties to be verified (see Lemma 4.3). This is a genuine breakthrough compared to [21, 24, 10, 2]. We believe this approach is still subject to extensions.

\section{Preliminaries}

In this section, we recall the main definitions for vector addition systems with states (VASS), without states (VAS) as well as the notions of reversal-boundedness introduced in [13, 8]. We also present the simultaneous unboundedness problem, which slightly generalizes place unboundedness problem for Petri 
nets. First, we write $\mathbb{N}$ [resp. $\mathbb{Z}]$ for the set of natural numbers [resp. integers] and $\left[m, m^{\prime}\right]$ with $m, m^{\prime} \in \mathbb{Z}$ to denote the set $\left\{j \in \mathbb{Z}: m \leq j \leq m^{\prime}\right\}$. For $\vec{x} \in \mathbb{Z}^{n}$, we write $\vec{x}(1), \ldots, \vec{x}(n)$ for the entries of $\vec{x}$. For $\vec{x}, \vec{y} \in \mathbb{Z}^{n}, \vec{x} \preceq \vec{y} \stackrel{\text { def }}{\Leftrightarrow}$ for $i \in[1, n]$, we have $\vec{x}(i) \leq \vec{y}(i)$. We also write $\vec{x} \prec \vec{y}$ when $\vec{x} \preceq \vec{y}$ and $\vec{x} \neq \vec{y}$.

\subsection{Simultaneous unboundedness problem for VASS}

VASS. A vector addition system with states [11] (VASS for short) is a finite-state automaton with transitions labelled by tuples of integers viewed as update functions. A VASS is a structure $\mathscr{V}=(Q, n, \delta)$ such that $Q$ is a nonempty finite set of control states, $n \geq 1$ is the dimension, and $\delta$ is the transition relation defined as a finite set of triples in $Q \times \mathbb{Z}^{n} \times Q$. Elements $t=\left(q, \vec{b}, q^{\prime}\right) \in \delta$ are called transitions and are often represented by $q \stackrel{\vec{b}}{\rightarrow} q^{\prime}$. VASS with a unique control state are called vector addition systems (VAS for short) [15]. In the sequel, a VAS $\mathscr{T}$ is represented by a finite nonempty subset of $\mathbb{Z}^{n}$, encoding naturally the transitions. VASS and VAS are equivalent to Petri nets, see e.g. [23]. In this paper, the decision problems are defined with the VASS model and the decision procedures are designed for VAS, assuming that we know how the problems can be reduced, see e.g. [11]. Indeed, we prefer to define problems with the help of the VASS model since when infinite-state transition systems arise in the modeling of computational processes, there is often a natural factoring of each system state into a control component and a memory component, where the set of control states is typically finite.

Runs. A configuration of $\mathscr{V}$ is defined as a pair $(q, \vec{x}) \in Q \times \mathbb{N}^{n}$ (for VAS, we simply omit the control state). An initialized VASS is a pair of a VASS and a configuration. Given two configurations $(q, \vec{x}),\left(q^{\prime}, \overrightarrow{x^{\prime}}\right)$ and a transition $t=q \stackrel{\vec{b}}{\rightarrow} q^{\prime}$, we write $(q, \vec{x}) \stackrel{t}{\rightarrow}\left(q^{\prime}, \overrightarrow{x^{\prime}}\right)$ whenever $\overrightarrow{x^{\prime}}=\vec{x}+\vec{b}$. We also write $(q, \vec{x}) \rightarrow\left(q^{\prime}, \overrightarrow{x^{\prime}}\right)$ when there is no need to specify the transition $t$. The operational semantics of VASS updates configurations, runs of such systems are essentially sequences of configurations. Every VASS induces a (possibly infinite) directed graph of configurations. Indeed, all the interesting problems on VASS can be formulated on its transition system $\left(Q \times \mathbb{N}^{n}, \rightarrow\right)$. Given a VASS $\mathscr{V}=(Q, n, \delta)$, a run $\rho$ is a nonempty (possibly infinite) sequence $\rho=\left(q_{0}, \vec{x}_{0}\right), \ldots,\left(q_{k}, \vec{x}_{k}\right), \ldots$ of configurations such that $\left(q_{i}, \vec{x}_{i}\right) \rightarrow$ $\left(q_{i+1}, \overrightarrow{x_{i+1}}\right)$ for all $i$. We set $\operatorname{Reach}\left(\mathscr{V},\left(q_{0}, \vec{x}_{0}\right)\right) \stackrel{\text { def }}{=}\left\{\left(q_{k}, \vec{x}_{k}\right):\right.$ there is a finite run $\left.\left(q_{0}, \vec{x}_{0}\right), \ldots,\left(q_{k}, \vec{x}_{k}\right)\right\}$. A path $\pi$ is a finite sequence of transitions whose successive control states respect $\delta$ (actually this notion is mainly used for VAS without control states). A pseudo-configuration is defined as an element of $Q \times \mathbb{Z}^{n}$. When $\pi=t_{1} \ldots t_{k}$ is a path, the pseudo-run $\rho=(\pi,(q, \vec{x}))$ is defined as the sequence of pseudoconfigurations $\left(q_{0}, \vec{x}_{0}\right) \cdots\left(q_{k}, \vec{x}_{k}\right)$ such that $\left(q_{0}, \vec{x}_{0}\right)=(q, \vec{x})$, and for $i \in[1, k]$, there is $t=q_{i} \stackrel{\vec{b}}{\rightarrow} q_{i+1}$ such that $\vec{x}_{i}=\vec{x}_{i-1}+\vec{b}$. So, we deliberately distinguish the notion of path (sequence of transitions) from the notion of pseudo-run (sequence of elements in $Q \times \mathbb{Z}^{n}$ respecting the transition from $\mathscr{V}$ ). We also use the notation $(q, \vec{x}) \stackrel{t}{\rightarrow}\left(q^{\prime}, \overrightarrow{x^{\prime}}\right)$ with pseudo-configurations. Given a VASS $\mathscr{V}$ [resp. a pseudo-configuration $(q, \vec{x})$, etc.] of dimension $n$, we write $\mathscr{V}(I)$ [resp. $(q, \vec{x})(I)$, etc.] to denote the restriction of $\mathscr{V}$ [resp. $(q, \vec{x})$, etc. $]$ to the components in $I \subseteq[1, n]$.

Sizes. Given $\vec{x} \in \mathbb{Z}^{n}$, we write $\operatorname{maxneg}(\vec{x})$ to denote the value $\max (\{\max (0,-\vec{x}(i)): i \in[1, n]\})$. By extension, we write $\operatorname{maxneg}(\mathscr{V})$ to denote $\max \left\{\operatorname{maxneg}(\vec{b}): q \stackrel{\vec{b}}{\rightarrow} q^{\prime} \in \delta\right\}$. Furthermore, we write $\operatorname{scale}(\mathscr{V})$ to denote the value $\max \left(\left\{|\vec{b}(i)|: q \stackrel{\vec{b}}{\rightarrow} q^{\prime} \in \delta, i \in[1, n]\right\}\right)$. For instance maxneg $((-2,3))=2$ and scale $(\{(-2,3)\})=3$. Given a VASS $\mathscr{V}=(Q, n, \delta)$, we write $|\mathscr{V}|$ to denote its size defined by $\operatorname{card}(Q)+n \times \operatorname{card}(\boldsymbol{\delta}) \times\left(2 \times \operatorname{card}(Q)+\left(2+\left\lceil\log _{2}(1+\operatorname{scale}(\mathscr{V}))\right\rceil\right)\right)$. Observe that $2+\left\lceil\log _{2}(1+a)\right\rceil$ is a sufficient number of bits to encode integers in $[-a, a]$ for $a>0$. Moreover scale $(\mathscr{V}) \geq \operatorname{maxneg}(\mathscr{V})$, $\operatorname{scale}(\mathscr{V}) \leq 2^{|\mathscr{V}|}$ and $|\mathscr{V}| \geq 2$

Simultaneous unboundedness problem. Let $\left(\mathscr{V},\left(q_{0}, \vec{x}_{0}\right)\right)$ be an initialized VASS of dimension $n$ and 
$X \subseteq[1, n]$. We say that $\left(\mathscr{V},\left(q_{0}, \vec{x}_{0}\right)\right)$ is simultaneously $X$-unbounded if for any $B \geq 0$, there is a run from $\left(q_{0}, \vec{x}_{0}\right)$ to $(q, \vec{y})$ such that for $i \in X$, we have $\vec{y}(i) \geq B$. When $X=\{j\}$, we say that $\left(\mathscr{V},\left(q_{0}, \vec{x}_{0}\right)\right)$ is $j$-unbounded. It is clear that $\left(\mathscr{V},\left(q_{0}, \vec{x}_{0}\right)\right)$ is bounded (i.e., $\operatorname{Reach}\left(\mathscr{V},\left(q_{0}, \vec{x}_{0}\right)\right)$ is finite) iff for all $j$, $\left(\mathscr{V},\left(q_{0}, \vec{x}_{0}\right)\right)$ is not $j$-unbounded. The simultaneous unboundedness problem is defined as follows: given an initialized VASS $\left(\mathscr{V},\left(q_{0}, \vec{x}_{0}\right)\right)$ of dimension $n$ and $X \subseteq[1, n]$, is $\left(\mathscr{V},\left(q_{0}, \vec{x}_{0}\right)\right)$ simultaneously $X$-unbounded?

Theorem 2.1. [15] Simultaneous unboundedness problem is decidable.

This follows from [15, 28]: $\left(\mathscr{V},\left(q_{0}, \vec{x}_{0}\right)\right)$ is simultaneously $X$-unbounded iff the coverability graph $C G\left(\mathscr{V},\left(q_{0}, \vec{x}_{0}\right)\right.$ ) (see e.g., [15, 28]) contains an extended configuration $(q, \vec{y})$ such that $\vec{y}(X)=\vec{\infty}$ (for $\alpha \in \mathbb{Z} \cup\{\infty\}$, we write $\vec{\alpha}$ to denote any vector of dimension $n \geq 1$ whose component values are $\alpha$ ).

\subsection{Standard reversal-boundedness and its new variant}

A reversal for a counter occurs in a run when there is an alternation from nonincreasing mode to nondecreasing mode and vice-versa. A VASS is reversal-bounded whenever there is $r \geq 0$ such that for any run, every counter makes no more than $r$ reversals. This class of VASS has been introduced and studied in [13], partly inspired by similar restrictions on multistack automata [3]. In spite of the fact that the problem of deciding whether a counter automaton (VASS with zero-tests) is reversal-bounded is undecidable [13], we shall see that reversal-bounded counter automata have numerous fundamental properties. Moreover, a breakthrough has been achieved in [8] by establishing that checking whether a VASS is reversal-bounded is decidable. The decidability proof in [8] provides a decision procedure that requires nonprimitive recursive time in the worst-case since Karp and Miller trees need to be built [15, 28]. Let $\mathscr{V}=(Q, n, \delta)$ be a VASS. Let us define the auxiliary VASS $\mathscr{V}_{r b}=\left(Q^{\prime}, 2 n, \boldsymbol{\delta}^{\prime}\right)$ such that essentially, the $n$ new components in $\mathscr{V}_{r b}$ count the number of reversals for each component from $\mathscr{V}$. We set $Q^{\prime}=Q \times\{\mathrm{DEC}, \mathrm{INC}\}^{n}$ and, for each $\vec{v} \in\{\mathrm{DEC}, \mathrm{INC}\}^{n}$ and $i \in[1, n], \vec{v}(i)$ encodes whether component $i$ is in a decreasing mode or in an increasing mode. Moreover, $(q$, mode $) \stackrel{\vec{b}^{\prime}}{\rightarrow}\left(q^{\prime}\right.$, mode $\left.e^{\prime}\right) \in \delta^{\prime}$ (with $\left.\overrightarrow{b^{\prime}} \in \mathbb{Z}^{2 n}\right) \stackrel{\text { def }}{\Leftrightarrow}$ there is $q \stackrel{\vec{b}}{\rightarrow} q^{\prime} \in \delta$ such that $\vec{b}^{\prime}([1, n])=\vec{b}$ and for every $i \in[1, n]$, one of the conditions below is satisfied:

- $\vec{b}(i)<0, \bmod e(i)=\bmod e^{\prime}(i)=\mathrm{DEC}$ and $\overrightarrow{b^{\prime}}(n+i)=0$,

- $\vec{b}(i)<0, \bmod e(i)=\mathrm{INC}, \operatorname{mode} e^{\prime}(i)=\mathrm{DEC}$ and $\vec{b}^{\prime}(n+i)=1$,

- $\vec{b}(i)>0, \operatorname{mode}(i)=\bmod e^{\prime}(i)=\mathrm{INC}$ and $\vec{b}^{\prime}(n+i)=0$,

- $\vec{b}(i)>0, \bmod e(i)=\mathrm{DEC}, \bmod e^{\prime}(i)=\mathrm{INC}$ and $\vec{b}^{\prime}(n+i)=1$,

- $\vec{b}(i)=0, \bmod e(i)=\operatorname{mode} e^{\prime}(i)$ and $\overrightarrow{b^{\prime}}(n+i)=0$.

Initialized VASS $(\mathscr{V},(q, \vec{x}))$ is reversal-bounded [13] $\stackrel{\text { def }}{\Leftrightarrow}$ for $i \in[n+1,2 n],\left\{\vec{y}(i): \exists \operatorname{run}\left(q_{r b}, \vec{x}_{r b}\right) \stackrel{*}{\rightarrow}\right.$ $\left(q^{\prime}, \vec{y}\right)$ in $\left.\mathscr{V}_{r b}\right\}$ is finite with $q_{r b}=(q, \mathrm{INC}), \vec{x}_{r b}$ restricted to the $n$ first components is $\vec{x}$ and $\vec{x}_{r b}$ restricted to the $n$ last components is $\overrightarrow{0}$. When $r \geq \max \left(\left\{\vec{y}(i): \exists \operatorname{run}\left(q_{r b}, \vec{x}_{r b}\right) \stackrel{*}{\rightarrow}\left(q^{\prime}, \vec{y}\right)\right.\right.$ in $\left.\mathscr{V}_{r b}\right\}: i \in$ $[n+1,2 n])(\mathscr{V},(q, \vec{x}))$ is said to be $r$-reversal-bounded. For $i \in[1, n]$, when $\left\{\vec{y}(n+i): \exists \operatorname{run}\left(q_{r b}, \vec{x}_{r b}\right) \stackrel{*}{\rightarrow}\right.$ $\left(q^{\prime}, \vec{y}\right)$ in $\left.\mathscr{V}_{r b}\right\}$ is finite, we say that $(\mathscr{V},(q, \vec{x}))$ is reversal-bounded with respect to $i$. A VASS $\mathscr{V}$ is globally reversal-bounded iff there is $r \geq 0$ such that for every configuration $(q, \vec{x}),(\mathscr{V},(q, \vec{x}))$ is $r$ reversal-bounded. Global reversal-boundedness detection can be easily reduced to reversal-boundedness detection. Indeed, it is sufficient to introduce a new control state $q_{\text {new }}$ that contains as many self-loops as the dimension $n$ and each self-loop $i$ increments the $i$ th component. Then, nondeterministically we jump 
to the rest of the VASS. In this way, $\left(\mathscr{V}^{\prime},\left(q_{\text {new }}, \overrightarrow{0}\right)\right)$ is reversal-bounded $\left(\mathscr{V}^{\prime}\right.$ is the new VASS obtained as a variant of $\mathscr{V}$ ) iff $\mathscr{V}$ is globally reversal-bounded (forthcoming upper bounds will apply to this problem too).

Reversal-boundedness for counter automata, and a fortiori for VASS, is very appealing because reachability sets are semilinear as recalled below.

Theorem 2.2. [13] Let $(\mathscr{V},(q, \vec{x}))$ be an $r$-reversal-bounded VASS. For each control state $q^{\prime}$, the set $\left\{\vec{y} \in \mathbb{N}^{n}: \exists \operatorname{run}(q, \vec{x}) \stackrel{*}{\rightarrow}\left(q^{\prime}, \vec{y}\right)\right\}$ is effectively semilinear.

This means that one can compute effectively a Presburger formula that characterizes precisely the reachable configurations whose control state is $q^{\prime}$. So, detecting reversal-boundedness for VASS, which can be easily reformulated as an unboundedness problem, is worth the effort since semilinearity follows and then decision procedures for Presburger arithmetic can be used.

Lemma 2.3. $(\mathscr{V},(q, \vec{x}))$ is reversal-bounded with respect to $i$ iff $\left(\mathscr{V}_{r b},\left(q_{r b}, \vec{x}_{r b}\right)\right)$ is not $(n+i)$-unbounded.

An interesting extension of reversal-boundedness is introduced in [8, 25] for which we only count the number of reversals when they occur for a counter value above a given bound $B$. For instance, finiteness of the reachability set implies reversal-boundedness in the sense of [8, 25], which we shall call weak reversal-boundedness. Let $\mathscr{V}=(Q, n, \delta)$ be a VASS and a bound $B \in \mathbb{N}$. Instead of defining a counter automaton $\mathscr{V}_{r b}$ as done to characterize (standard) reversal-boundedness, we define directly an infinite directed graph that corresponds to a variant of the transition system of $\mathscr{V}_{r b}$ : still, there are $n$ new counters that record the number of reversals but only if they occur above a bound $B$. That is why, the infinite directed graph $T S_{B}=\left(Q \times\{\mathrm{DEC}, \mathrm{INC}\}^{n} \times \mathbb{N}^{2 n}, \rightarrow_{B}\right)$ is defined as follows: $(q, \operatorname{mode}, \vec{x}) \rightarrow_{B}\left(q^{\prime}, \operatorname{mode}^{\prime}, \vec{x}^{\prime}\right)$ $\stackrel{\text { def }}{\Leftrightarrow}$ there is a transition $q \stackrel{\vec{b}}{\rightarrow} q^{\prime} \in \delta$ such that $\vec{x}^{\prime}([1, n])=\vec{x}([1, n])+\vec{b}$, and for every $i \in[1, n]$, one of the conditions below is satisfied:

- $\vec{b}(i)<0, \bmod e(i)=\bmod e^{\prime}(i)=\mathrm{DEC}$ and $\overrightarrow{b^{\prime}}(n+i)=0$,

- $\vec{b}(i)<0, \bmod e(i)=\mathrm{INC}, \bmod e^{\prime}(i)=\mathrm{DEC}, \vec{x}(i) \leq B$ and $\overrightarrow{b^{\prime}}(n+i)=0$,

- $\vec{b}(i)<0, \bmod e(i)=\mathrm{INC}, \bmod e^{\prime}(i)=\mathrm{DEC}, \vec{x}(i)>B$ and $\overrightarrow{b^{\prime}}(n+i)=1$,

- $\vec{b}(i)>0, \operatorname{mode}(i)=\bmod e^{\prime}(i)=\mathrm{INC}$ and $\vec{b}^{\prime}(n+i)=0$,

- $\vec{b}(i)>0, \bmod e(i)=\mathrm{DEC}, \bmod e^{\prime}(i)=\mathrm{INC}, \vec{x}(i)>B$ and $\overrightarrow{b^{\prime}}(n+i)=1$,

- $\vec{b}(i)>0, \bmod e(i)=\mathrm{DEC}, \bmod e^{\prime}(i)=\mathrm{INC}, \vec{x}(i) \leq B$ and $\vec{b}^{\prime}(n+i)=0$,

- $\vec{b}(i)=0, \bmod e(i)=\overrightarrow{m o d e} e^{\prime}(i)$ and $\vec{b}^{\prime}(n+i)=0$.

Initialized VASS $(\mathscr{V},(q, \vec{x}))$ is weakly reversal-bounded $[8] \stackrel{\text { def }}{\Leftrightarrow}$ there is some $B \geq 0$ such that for $i \in[n+$ $1,2 n],\left\{\vec{y}(i):\left(q_{r b}, \vec{x}_{r b}\right) \stackrel{*}{\rightarrow}_{B}\left(q^{\prime}, \vec{y}\right)\right.$ in $\left.T S_{B}\right\}$ is finite. When $r \geq \max \left(\left\{\vec{y}(i):\left(q_{r b}, \vec{x}_{r b}\right) \stackrel{*}{\rightarrow}_{B}\left(q^{\prime}, \vec{y}\right)\right.\right.$ in $\left.T S_{B}\right\}:$ $i \in[n+1,2 n])(\mathscr{V},(q, \vec{x}))$ is said to be $r$-reversal-B-bounded. Observe that whenever $(\mathscr{V},(q, \vec{x}))$ is $r$ reversal-bounded, $(\mathscr{V},(q, \vec{x}))$ is $r$-reversal-0-bounded. As shown in [8], $r$-reversal- $B$-boundedness for some known $r$ and $B$ also leads to effective semilinearity of reachability sets and therefore detecting weak reversal-boundedness is also worth the effort. The reversal-boundedness detection problem is defined as follows: given an initialized VASS $(\mathscr{V},(q, \vec{x}))$ of dimension $n$ and $i \in[1, n]$, is $(\mathscr{V},(q, \vec{x}))$ reversal-bounded with respect to the component $i$ ? We also consider the variant with weak reversalboundedness.

Let us conclude this section by Lemma 2.4 below. The proof is essentially based on [11, Lemma 2.1] and on the definition of the initialized VASS $\left(\mathscr{V}_{r b},\left(q_{r b}, \vec{x}_{r b}\right)\right)$. The key properties are that the dimension increases only linearly and the scale "only" exponentially in the dimension. 
Lemma 2.4. Given $\mathscr{V}=(Q, n, \delta)$ and a configuration $(q, \vec{x})$, one can effectively build in polynomial space an initialized VAS $\left(\mathscr{T}, \vec{x}^{\prime}\right)$ of dimension $2 n+3$ such that $(\mathscr{V},(q, \vec{x}))$ is reversal-bounded with respect to $i$ iff $\left(\mathscr{T}, \vec{x}^{\prime}\right)$ is not $(n+i)$-unbounded. Moreover, $\operatorname{scale}(\mathscr{T})=\max \left(\left(\operatorname{card}(Q) \times 2^{n}+1\right)^{2}, \operatorname{scale}(\mathscr{V})\right)$.

Note also that by using the simple reduction from VASS to VAS that increases the dimension by the number of control states, we would increase exponentially the dimension, which would disallow us to obtain forthcoming optimal complexity bounds. In Lemma 3.5, we shall explain how to reduce weak reversal-boundedness detection to a generalization of $(n+i)$-unboundedness.

\section{Generalized Unboundedness Properties}

In this section, we essentially introduce the generalized unboundedness problem and we show how several detection problems can be naturally reduced to it.

\subsection{Witness runs for simultaneous unboundedness}

We know that $\left(\mathscr{V},\left(q_{0}, \vec{x}_{0}\right)\right)$ is $i$-unbounded iff the coverability graph $C G\left(\mathscr{V},\left(q_{0}, \vec{x}_{0}\right)\right)$ (see e.g., [15, 28]) contains an extended configuration with $\infty$ on the ith component. This is a simple characterization whose main disadvantage is to induce a nonprimitive recursive decision procedure in the worst-case. By contrast, unboundedness of $\left(\mathscr{V},\left(q_{0}, \vec{x}_{0}\right)\right)$ (i.e. $i$-unboundedness for some $\left.i \in[1, n]\right)$ is equivalent to the existence of witness run of the form $\left(q_{0}, \vec{x}_{0}\right) \stackrel{*}{\rightarrow}\left(q_{1}, \vec{x}_{1}\right) \stackrel{+}{\rightarrow}\left(q_{2}, \vec{x}_{2}\right)$ such that $\vec{x}_{1} \prec \vec{x}_{2}$ and $q_{1}=q_{2}$. In [21], it is shown that if there is such a run, there is one of length at most doubly exponential. Given a component $i \in[1, n]$, a natural adaptation to $i$-unboundedness is to check the existence of a run of the form $\left(q_{0}, \vec{x}_{0}\right) \stackrel{*}{\rightarrow}\left(q_{1}, \vec{x}_{1}\right) \stackrel{\pi}{\rightarrow}\left(q_{2}, \vec{x}_{2}\right)$ such that $\vec{x}_{1} \prec \vec{x}_{2}, q_{1}=q_{2}$ and $\vec{x}_{1}(i)<\vec{x}_{2}(i)$. By inspecting the proof in [21], one can show that if there is such a run, then there is one of length at most doubly exponential. However, although existence of such a run is a sufficient condition for $i$-unboundedness (simply iterate $\pi$ infinitely), this is not necessary as shown on the VASS below:

$$
\left(\begin{array}{l}
1 \\
0
\end{array}\right) \quad A \quad \begin{gathered}
\left(\begin{array}{l}
0 \\
0
\end{array}\right) \\
\end{gathered} \quad B \quad\left(\begin{array}{c}
-1 \\
1
\end{array}\right)
$$

The second component is unbounded from $(A, \overrightarrow{0})$ but no run $(A, \overrightarrow{0}) \stackrel{*}{\rightarrow}\left(q, \overrightarrow{x_{1}}\right) \stackrel{\pi}{\rightarrow}\left(q, \overrightarrow{x_{2}}\right)$ with $\overrightarrow{x_{1}} \prec \overrightarrow{x_{2}}$, $\vec{x}_{1}(2)<\vec{x}_{2}(2)$ and $q \in\{A, B\}$ exists. Indeed, in order to increment the second component, the first component needs first to be incremented. The ultimate condition for simultaneous unboundedness needs to specify the different ways to introduce the value $\infty$ along a given branch of the Karp and Miller trees. This is done thanks to the condition $\mathrm{PB}_{\sigma}$ defined below and generalized in Section 3.2. A disjointness sequence is a nonempty sequence $\sigma=X_{1} \cdots X_{K}$ of nonempty subsets of $[1, n]$ such that for $i \neq i^{\prime}$, $X_{i} \cap X_{i^{\prime}}=\emptyset$ (consequently $\left.K \leq n\right)$. A run of the form

$$
\left(q_{0}, \vec{x}_{0}\right) \stackrel{\pi_{0}^{\prime}}{\rightarrow}\left(q_{1}, \vec{x}_{1}\right) \stackrel{\pi_{1}}{\rightarrow}\left(q_{2}, \vec{x}_{2}\right) \stackrel{\pi_{1}^{\prime}}{\rightarrow} \cdots \stackrel{\pi_{K-1}^{\prime}}{\rightarrow}\left(q_{2 K-1}, \vec{x}_{2 K-1}\right) \stackrel{\pi_{K}}{\rightarrow}\left(q_{2 K}, \vec{x}_{2 K}\right)
$$

satisfies the property $\mathrm{PB}_{\sigma}$ (Place Boundedness with respect to a disjointness sequence $\sigma$ ) iff the conditions below hold true:

(P0) For $l \in[1, K], q_{2 l-1}=q_{2 l}$.

(STRICT) for $l \in[1, K]$ and $j \in X_{l}, x_{2 l-1}(j)<\overrightarrow{x_{2 l}}(j)$.

(NONSTRICT) For $l \in[1, K]$ and $j \in\left([1, n] \backslash X_{l}\right), \overrightarrow{x_{2 l}}(j)<x_{2 l-1}(j)$ implies $j \in \bigcup_{l^{\prime} \in[1, l-1]} X_{l^{\prime}}$. 
Observe that when (STRICT) holds, the condition (NONSTRICT) is equivalent to: for all $l \in[1, K]$ and all $j \notin \underset{l^{\prime} \in[1, l-1]}{\bigcup} X_{l^{\prime}}$, we have $\vec{x}_{2 l-1}(j) \leq \vec{x}_{2 l}(j)$. Consequently, for all $l \in[1, K]$ and all paths of the form $\left(\pi_{l}\right)^{k}$ for some $k \geq 1$, the effect on the $j$ th component may be negative only if $j \in \underset{l^{\prime} \in[1, l-1]}{\bigcup} X_{l^{\prime}}$. It is now time to provide a witness run characterization for simultaneous $X$-unboundedness that is a direct consequence of the properties of the coverability graphs [28].

Lemma 3.1. Let $\left(\mathscr{V},\left(q_{0}, \vec{x}_{0}\right)\right)$ be an initialized VASS of dimension $n$ and $X \subseteq[1, n]$. Then, $\left(\mathscr{V},\left(q_{0}, \vec{x}_{0}\right)\right)$ is simultaneously $X$-unbounded iff there is a run $\rho$ starting at $\left(q_{0}, \vec{x}_{0}\right)$ satisfying $\mathrm{PB}_{\sigma}$ for some disjointness sequence $\sigma=X_{1} \cdots X_{K}$ such that $X \subseteq\left(X_{1} \cup \cdots \cup X_{K}\right)$ and $X \cap X_{K} \neq \emptyset$.

Consequently, $\left(\mathscr{V},\left(q_{0}, \vec{x}_{0}\right)\right)$ is $i$-unbounded iff there is a run $\rho$ starting at $\left(q_{0}, \vec{x}_{0}\right)$ satisfying $\mathrm{PB}_{\sigma}$ for some disjointness sequence $\sigma=X_{1} \cdots X_{K}$ with $i \in X_{K}$. This can be expressed in the logical formalisms from [29, 2] but this requires a formula of exponential size in the dimension because an exponential number of disjointness sequences needs to be taken into account. By contrast, each disjunct has only polynomial-size in $n$. The path formula looks like that:

$$
\bigvee_{X_{1} \cdots X_{K}, i \in X_{K}} \exists \vec{x}_{1}, \ldots, \vec{x}_{2 K} \bigwedge_{l=1}^{K}\left(\bigwedge_{j \in X_{l}} \vec{x}_{2 l-1}(j)<\vec{x}_{2 l}(j)\right) \wedge\left(\bigwedge_{j \notin\left(X_{1} \cup \ldots \cup X_{l-1}\right)}^{\left.\bigwedge \vec{x}_{2 l-1}(j) \leq \vec{x}_{2 l}(j)\right)}\right.
$$

It is worth noting that the satisfaction of $\mathrm{PB}_{\sigma}$ does not imply $\vec{x}_{1} \preceq \vec{x}_{2 K}$. This prevents us from defining this condition with an increasing path formula [2] and therefore the EXPSPACE upper bound established in [2] does not apply directly to $i$-unboundedness.

\subsection{A helpful generalization}

We introduce below a slight generalization of the above properties $\mathrm{PB}_{\sigma}$ in order to underline their essential features and to provide a uniform treatment. Moreover, this will allow us to express new properties, for instance for regularity detection. The conditions (STRICT) and (NONSTRICT) specify inequality constraints between component values. We introduce intervals in place of such constraints. An interval is an expression of one of the forms $]-\infty,+\infty[,[a,+\infty[]-,\infty, b]$ or $[a, b]$ for some $a, b \in \mathbb{Z}$ (with the obvious interpretation). A generalized unboundedness property $\mathscr{P}=\left(\mathscr{I}_{1}, \ldots, \mathscr{I}_{K}\right)$ is a nonempty sequence of $n$-tuples of intervals. The length of $\mathscr{P}$ is $K$ and its scale is equal to the maximum between 1 and the maximal absolute value of integers occurring in the interval expressions of $\mathscr{P}$ (if any). A run of the form $\left(q_{0}, \vec{x}_{0}\right) \stackrel{\pi_{0}^{\prime}}{\rightarrow}\left(q_{1}, \vec{x}_{1}\right) \stackrel{\pi_{1}}{\rightarrow}\left(q_{2}, \vec{x}_{2}\right) \stackrel{\pi_{1}^{\prime}}{\rightarrow}\left(q_{3}, \vec{x}_{3}\right) \cdots \stackrel{\pi_{K-1}^{\prime}}{\longrightarrow}\left(q_{2 K-1}, \vec{x}_{2 K-1}\right) \stackrel{\pi_{K}}{\rightarrow}\left(q_{2 K}, \vec{x}_{2 K}\right)$ satisfies the property $\mathscr{P} \stackrel{\text { def }}{\Leftrightarrow}(\mathrm{P} 0)$ and the conditions below hold true:

(P1) For $l \in[1, K]$ and $j \in[1, n]$, we have $\vec{x}_{2 l}(j)-\vec{x}_{2 l-1}(j) \in \mathscr{I}_{l}(j)$.

(P2) For $l \in[1, K]$ and $j \in[1, n]$, if $\overrightarrow{x_{2 l}}(j)-x_{2 l-1}(j)<0$, then there is $l^{\prime}<l$ s.t. $\vec{x}_{2 l^{\prime}}(j)-\vec{x}_{2 l^{\prime}-1}(j)>0$.

Given a run $\rho$, we say that it satisfies $\mathscr{P}$ if it admits a decomposition satisfying the adequate conditions. By extension, $\left(\mathscr{V},\left(q_{0}, \vec{x}_{0}\right)\right)$ satisfies $\mathscr{P} \stackrel{\text { def }}{\Leftrightarrow}$ there is a finite run starting at $\left(q_{0}, \vec{x}_{0}\right)$ satisfying $\mathscr{P}$. It is easy to see that condition (P1) [resp. (P2)] is a quantitative counterpart for condition (STRICT) [resp. (NONSTRICT)]. The generalized unboundedness problem is defined as follows: given an initialized $\operatorname{VASS}\left(\mathscr{V},\left(q_{0}, \vec{x}_{0}\right)\right)$ and a generalized unboundedness property $\mathscr{P}$, does $\left(\mathscr{V},\left(q_{0}, \vec{x}_{0}\right)\right)$ satisfy $\mathscr{P}$ ? Let us first forget about control states: we can safely restrict ourselves to VAS without any loss of generality, as it is already the case for many properties. 
Lemma 3.2. There is a logspace many-one reduction from the generalized unboundedness problem for VASS to the generalized unboundedness problem for VAS. Moreover, an instance $((\mathscr{V},(q, \vec{x})), \mathscr{P})$ is reduced to an instance $\left(\left(\mathscr{T}, \vec{x}^{\prime}\right), \mathscr{P}^{\prime}\right)$ such that (1) if $\mathscr{V}$ is of dimension $n$, then $\mathscr{T}$ is of dimension $n+3$, (2) $\mathscr{P}$ and $\mathscr{P}^{\prime}$ have the same length and scale and (3) scale $(\mathscr{T})=\max \left((\operatorname{card}(Q)+1)^{2}, \operatorname{scale}(\mathscr{V})\right)$ where $Q$ is the set of control states of $\mathscr{V}$.

The proof is essentially based on [11, Lemma 2.1]. Generalized unboundedness properties can be expressed in even more general formalisms for which decidability is known. However, in Section 4 , we shall establish EXPSPACE-completeness.

Theorem 3.3. [2] The generalized unboundedness problem is decidable.

Given $\left(\mathscr{V},\left(q_{0}, \vec{x}_{0}\right)\right)$, the existence of a run from $\left(q_{0}, \overrightarrow{x_{0}}\right)$ satisfying $\mathscr{P}$ can be easily expressed in Yen's path logic [29] and the generalized unboundedness problem is therefore decidable by [2, Theorem 3] and [18, 16]. We cannot rely on [29, Theorem 3.8] for decidability since [29, Lemma 3.7] contains a flaw, as observed in [2]. [2] precisely establishes that satisfiability in Yen's path logic is equivalent to the reachability problem for VASS. Moreover, it is worth noting that the reduction from the reachability problem to satisfiability [2. Theorem 2] uses path formulae that cannot be expressed as generalized unboundedness properties. Observe that the EXPSPACE upper bound obtained for increasing path formulae in [2, Section 6] cannot be used herein since obviously generalized unboundedness properties are not necessarily increasing. That is why, we need directly to extend Rackoff's proof for boundedness [21].

\subsection{From regularity to reversal-boundedness detection}

In this section, we briefly explain how simultaneous unboundedness problem, regularity detection, strong promptness detection and weak reversal-boundness detection can be reduced to generalized unboundedness problem. This will allow us to obtain EXPSPACE upper bound for all these problems.

Simultaneous unboundedness problem. It is easy to show that every property $\mathrm{PB}_{\sigma}$ can be encoded as a generalized unboundedness property $\mathscr{P}_{\sigma}$ with length $K \leq n$ and scale $\left(\mathscr{P}_{\sigma}\right)=1$. Indeed, from a disjointness sequence $\sigma=X_{1} \cdots X_{K}$, we define $\mathscr{P}_{\sigma}=\left(\mathscr{I}_{1}, \ldots, \mathscr{I}_{K}\right)$ as follows. For $l \in[1, K]$ and $j \in[1, n]$, if $j \in X_{l}$ then $\mathscr{I}_{l}(j)=\left[1,+\infty\left[\right.\right.$. Otherwise, if $j \in\left([1, n] \backslash\left(\bigcup_{1 \leq l^{\prime} \leq l} X_{l^{\prime}}\right)\right)$, then $\mathscr{I}_{l}(j)=[0,+\infty[$, otherwise $\left.\mathscr{I}_{l}(j)=\right]-\infty,+\infty\left[\right.$. It is then easy to check that $\sigma$ and $\mathrm{PB}_{\sigma}$ define the same set of runs.

Regularity detection. Another example of properties that can be encoded by generalized unboundedness properties comes from the witness run characterization for nonregularity, see e.g. [28, 2]. Nonregularity of an initialized VASS $\left(\mathscr{V},\left(q_{0}, \overrightarrow{x_{0}}\right)\right)$ is equivalent to the existence of a run of the form $\left(q_{0}, \overrightarrow{x_{0}}\right) \stackrel{\pi_{0}^{\prime}}{\rightarrow}$ $\left(q_{1}, \overrightarrow{x_{1}}\right) \stackrel{\pi_{1}}{\rightarrow}\left(q_{2}, \overrightarrow{x_{2}}\right) \stackrel{\pi_{1}^{\prime}}{\rightarrow}\left(q_{3}, \overrightarrow{x_{3}}\right) \stackrel{\pi_{2}}{\rightarrow}\left(q_{4}, \overrightarrow{x_{4}}\right)$ such that $q_{1}=q_{2}, q_{3}=q_{4}$, there is $i \in[1, n]$ such that $\overrightarrow{x_{1}} \prec \overrightarrow{x_{2}}$, $\overrightarrow{x_{4}}(i)<\overrightarrow{x_{3}}(i)$ and for all $j \in[1, n]$ such that $\overrightarrow{x_{4}}(j)<\overrightarrow{x_{3}}(j)$, we have $\overrightarrow{x_{1}}(j)<\overrightarrow{x_{2}}(j)$, see e.g. [28, 2]. Consequently, nonregularity condition can be viewed as a disjunction of generalized unboundedness properties of the form $\left(\mathscr{I}_{1}^{i}, \mathscr{I}_{2}^{i}\right)$ where $\mathscr{I}_{1}^{i}(i)=\left[1,+\infty\left[, \mathscr{I}_{2}^{i}(i)=\right]-\infty,-1\right]$, and for $j \neq i$, we have $\mathscr{I}_{1}^{i}(j)=[0,+\infty[$ and $\left.\mathscr{I}_{2}^{i}(j)=\right]-\infty,+\infty[$.

Strong promptness detection. We show below how the strong promptness detection problem can be reduced to the simultaneous unboundedness problem, leading to an EXPSPACE upper bound. The strong promptness detection problem is defined as follows [27]: given $((Q, n, \delta),(q, \vec{x}))$ and a partition $\left(\boldsymbol{\delta}_{I}, \boldsymbol{\delta}_{E}\right)$ 
of $\delta$, is there $B \in \mathbb{N}$ such that for every run $(q, \vec{x}) \stackrel{*}{\rightarrow}\left(q^{\prime}, \vec{x}^{\prime}\right)$, there is no run $\left(q^{\prime}, \vec{x}^{\prime}\right) \stackrel{\pi}{\rightarrow}\left(q^{\prime \prime}, \vec{x}^{\prime \prime}\right)$ using only transitions from $\delta_{I}$ and of length more than $B\left(\pi \in \delta_{I}^{*}\right)$ ? The transitions in $\delta_{I}$ are called internal and strong promptness guarantees that sequences of internal transitions cannot be arbitrarily long. Let us consider below the VASS $\mathscr{V}$ of dimension 1 with $\delta_{I}$ made of the two transitions in bold.

$$
+1 \quad A \quad 0 \quad B^{-1} \quad C
$$

$(\mathscr{V},(A, 0))$ is not strongly prompt and there is no $(A, 0) \stackrel{*}{\rightarrow} \overline{(q,} \vec{x}) \stackrel{\pi}{\rightarrow}(q, \vec{y})$ for some $q \in\{A, B, C\}$ such that $\vec{x} \preceq \vec{y}, \pi$ is nonempty and contains only transitions in $\delta_{I}$.

Lemma 3.4. There is a logspace reduction from strong promptness detection problem to the complement of simultaneous unboundedness problem.

Weak reversal-boundedness detection. Complement of weak reversal-boundedness involves two universal quantifications (on $B$ and $r$ ) that can be understood as simultaneous unboundedness properties. Lemma 3.5 below is a key intermediate result in our investigation.

Lemma 3.5. Given a VASS $\mathscr{V}=(Q, n, \delta)$ and a configuration $(q, \vec{x}),(\mathscr{V},(q, \vec{x}))$ is not weakly reversalbounded with respect to $i$ iff $\left(\mathscr{V}_{r b},\left(q_{r b}, \vec{x}_{r b}\right)\right)$ has a run satisfying $\mathrm{PB}_{\sigma}$ for some disjointness sequence $\sigma=X_{1} \cdots X_{K}$ with $n+i \in X_{K}$ and $i \in\left(X_{1} \cup \cdots \cup X_{K-1}\right)$.

As a corollary, we are in a position to present a witness run characterization for weak reversal-boundedness detection. $\left(\mathscr{V},\left(q_{0}, \vec{x}_{0}\right)\right)$ is not weakly reversal-bounded with respect to $i$ iff there exist a disjointness sequence $\sigma=X_{1} \cdots X_{K}$ and a run $\left(q_{0}, \vec{x}_{0}\right) \stackrel{\pi_{0}^{\prime}}{\rightarrow}\left(q_{1}, \vec{x}_{1}\right) \stackrel{\pi_{1}}{\rightarrow}\left(q_{2}, \vec{x}_{2}\right) \stackrel{\pi_{1}^{\prime}}{\rightarrow} \cdots \stackrel{\pi_{K}^{\prime}}{\rightarrow}\left(q_{2 K+1}, \vec{x}_{2 K+1}\right) \stackrel{\pi_{K+1}}{\rightarrow}$ $\left(q_{2 K+2}, \vec{x}_{2 K+2}\right)$ such that (1) $\pi_{K+1}$ contains a reversal for the $i$ th component, (2) the subrun $\left(q_{0}, \vec{x}_{0}\right) \stackrel{*}{\rightarrow}$ $\left(q_{2 K}, \vec{x}_{2 K}\right)$ satisfies $\mathrm{PB}_{\sigma},(3) i \in\left(X_{1} \cup \cdots \cup X_{K}\right)$ and (4) for $j \in[1, n], \vec{x}_{2 K+2}(j)<\vec{x}_{2 K+1}(j)$ implies $j \in\left(X_{1} \cup \cdots \cup X_{K}\right)$. Based on Lemmas 2.3 and 3.1, a characterization for reversal-boundedness can be also defined.

\subsection{A first relaxation}

Below, we relax the satisfaction of the property $\mathscr{P}$ by allowing negative component values in a controlled way. A pseudo-run of the form $\left(q_{0}, \vec{x}_{0}\right) \stackrel{\pi_{0}^{\prime}}{\rightarrow}\left(q_{1}, \vec{x}_{1}\right) \stackrel{\pi_{1}}{\rightarrow}\left(q_{2}, \vec{x}_{2}\right) \stackrel{\pi_{1}^{\prime}}{\rightarrow}\left(q_{3}, \vec{x}_{3}\right) \cdots \stackrel{\pi_{K-1}^{\prime}}{\rightarrow}\left(q_{2 K-1}, \vec{x}_{2 K-1}\right) \stackrel{\pi_{K}}{\rightarrow}$ $\left(q_{2 K}, \vec{x}_{2 K}\right)$ weakly satisfies $\mathscr{P} \stackrel{\text { def }}{\Leftrightarrow}$ it satisfies (P0), (P1), (P2) and (P3) defined as follows: for $j \in[1, n]$, every pseudo-configuration $\vec{x}$ such that $\vec{x}(j)<0$ occurs after some $\vec{x}_{2 l}$ for which $\vec{x}_{2 l}(j)-\vec{x}_{2 l-1}(j)>0$. If the run $\rho$ satisfies $\mathscr{P}$, then viewed as a pseudo-run, it also weakly satisfies $\mathscr{P}$. Lemma 3.6 below states that the existence of pseudo-runs weakly satisfying $\mathscr{P}$ is equivalent to the existence of runs satisfying $\mathscr{P}$ and their length can be compared. Later, we shall use the witness pseudo-run characterization.

Lemma 3.6. Let $\rho$ be a pseudo-run of length $L$ weakly satisfying $\mathscr{P}$ (of length $K$ ). Then, there is a run $\rho$ satisfying $\mathscr{P}$ of length at most $\left((L \times \operatorname{maxneg}(\mathscr{V}))^{K} \times\left(1+K^{2} \times L \times \operatorname{maxneg}(\mathscr{V})\right)+L\right.$.

The principle of the proof of Lemma 3.6 (and part of the proof of Lemma 3.1) is identical to the idea of the proof of the following property of the coverability graph $C G\left(\mathscr{V},\left(q_{0}, \overrightarrow{x_{0}}\right)\right)$ (see e.g., details in [23]). For every extended configuration $\left(q, \overrightarrow{y^{\prime}}\right) \in Q \times(\mathbb{N} \cup\{\infty\})^{n}$ in $C G\left(\mathscr{V},\left(q_{0}, \overrightarrow{x_{0}}\right)\right)$ and bound $B \in \mathbb{N}$, there is a run $\left(q_{0}, \overrightarrow{x_{0}}\right) \stackrel{*}{\rightarrow}(q, \vec{y})$ in $\mathscr{V}$ such that for $i \in[1, n]$, if $\overrightarrow{y^{\prime}}(i)=\infty$ then $\vec{y}(i) \geq B$ otherwise $\vec{y}(i)=\overrightarrow{y^{\prime}}(i)$. In the proof of Lemma 3.6, the paths $\pi_{i}$ 's are repeated hierarchically in order to eliminate negative values. Additionally, if $\rho$ is a pseudo-run of length $L$ weakly satisfying $\mathscr{P}$ and $L$ is at most doubly exponential in $N=|\mathscr{V}|+\left|\left(q_{0}, \vec{x}_{0}\right)\right|+K+\operatorname{scale}(\mathscr{P})$, then there is a run satisfying $\mathscr{P}$ and starting in $\vec{x}_{0}$ that is also of length at most doubly exponential in $N$. 


\section{EXPSPACE Upper Bound}

In this section, we deal with VAS only and we consider a current VAS $\mathscr{T}$ of dimension $n$ (see Lemma3.2). W.1.o.g., we can assume that $n>1$, otherwise it is easy to show that the generalized unboundedness problem restricted to VAS of dimension 1 can be solved in polynomial space. Moreover, we assume that $\operatorname{maxneg}(\mathscr{T}) \geq 1$.

\subsection{Approximating generalized unboundedness properties}

Generalized unboundedness properties apply on runs but as it will be shown below, it would be more convenient to relax the conditions to pseudo-runs. A first step has been done in Section 3.4, we shall push further the idea in order to adapt Rackoff's proof. Let $\rho=\vec{x}_{0} \stackrel{\pi_{0}^{\prime}}{\rightarrow} \vec{x}_{1} \stackrel{\pi_{1}}{\rightarrow} \vec{x}_{2} \cdots \vec{x}_{2 K-1} \stackrel{\pi_{K}}{\rightarrow} \vec{x}_{2 K}$ be a pseudo-run weakly satisfying $\mathscr{P}=\left(\mathscr{I}_{1}, \ldots, \mathscr{I}_{K}\right)$. We suppose that $\rho$ is induced by the path $t_{1} \ldots t_{k}$ with $\rho=\vec{u}_{0} \cdots \vec{u}_{k}$ and $f:[0,2 K] \rightarrow[0, k]$ is the map such that $\vec{x}_{i}=\vec{u}_{f(i)}(f(0)=0, f(2 K)=k)$. For each position $j \in[0, f(2 K-2)]$ along $\rho$, there is a maximal $l_{j} \in[1, K]$ (with respect to standard ordering on $\mathbb{N}$ ) and $\operatorname{INCR}_{j} \subseteq[1, n]$ such that $f\left(2 l_{j}-2\right) \leq j$ and $\operatorname{INCR}_{j}=\left\{i \in[1, n]: \exists l^{\prime} \in\left[1, l_{j}-1\right]\right.$ such that $x_{2 l^{\prime}-1}(i)<$ $\left.\overrightarrow{x_{2} l^{\prime}}(i)\right\}$. In the induction proof of Lemma 4.3 , we will need to check properties on suffixes of pseudoruns and it will be useful to approximate $\mathscr{P}$ with respect to some suffix $\left(\mathscr{I}_{l_{j}}, \ldots, \mathscr{I}_{K}\right)$ and to some set of components $\mathrm{INCR}_{j}$. Indeed, the suffix $\vec{u}_{l_{j}} \cdots \vec{u}_{k}$ weakly satisfies $\left(\mathscr{I}_{l_{j}}, \ldots, \mathscr{I}_{K}\right)$ assuming that we know how to increment strictly the components from $\mathrm{INCR}_{j}$. Moreover, like the notion of $i$ - $B$-boundedness from [21], we would like to enforce that for each component $j$ from a given set $I$ and for each pseudoconfiguration $\vec{y}$ along the pseudo-run satisfying the approximation property, either $\vec{y}(j)$ belongs to $[0, B-$ 1] or the prefix pseudo-run terminating on $\vec{y}$ has the ability to increase arbitrarily the value $\vec{y}(j)$ (this will correspond to condition (P2') below). So, we are now in position to define the approximation property $\mathscr{A}[\mathscr{P}, l, \mathrm{INCR}, I, B]$. Given a generalized unboundedness property $\mathscr{P}$ of length $K, l \in[1, K]$, INCR $\subseteq[1, n], I \subseteq[1, n]$ and $B \geq 0$, a pseudo-run of the form below

$$
\vec{y}_{2 l-2} \stackrel{\pi_{l-1}^{\prime}}{\longrightarrow} \vec{y}_{2 l-1} \stackrel{\pi_{l}}{\rightarrow} \vec{y}_{2 l} \cdots \stackrel{\pi_{K-1}^{\prime}}{\longrightarrow} \vec{y}_{2 K-1} \stackrel{\pi_{K}}{\rightarrow} \vec{y}_{2 K}
$$

satisfies the approximation property $\mathscr{A}[\mathscr{P}, l, \mathrm{INCR}, I, B]$ (also abbreviated by $\mathscr{A}$ ) $\stackrel{\text { def }}{\Leftrightarrow}$ the conditions below are verified:

(P1') For $l^{\prime} \in[l, K]$ and $j \in[1, n]$, we have $\vec{y}_{2 l^{\prime}}(j)-\vec{y}_{2 l^{\prime}-1}(j) \in \mathscr{I}_{l^{\prime}}(j)$.

$\left(\mathbf{P 2}^{\prime}\right)$ For $l^{\prime} \in[l, K]$ and $j \in[1, n]$, if $\vec{y}_{2 l^{\prime}}(j)-\vec{y}_{2 l^{\prime}-1}(j)<0$, then (there is $l \leq l^{\prime \prime}<l^{\prime}$ such that $\vec{x}_{2 l^{\prime \prime}}(j)-$ $\vec{x}_{2 l^{\prime \prime}-1}(j)>0$ or $j \in$ INCR).

$\left(\mathbf{P 3}^{\prime}\right)$ For every pseudo-configuration $\vec{x}$ in $\rho$ occurring between $\vec{y}_{2 l^{\prime}}$ and strictly before $\vec{y}_{2 l^{\prime}+2}$ with $l^{\prime} \geq$ $l-1, \vec{x}(J) \in[0, B-1]^{J}$ with $J=I \backslash\left(\operatorname{INCR} \cup\left\{j: \exists l \leq l^{\prime \prime} \leq l^{\prime}, \vec{x}_{2 l^{\prime \prime}}(j)-\vec{x}_{2 l^{\prime \prime}-1}(j)>0\right\}\right)$.

Condition ( $\left.\mathrm{P}^{\prime}\right)$ reflects the intuition that only the values from components in $J$ require to be controlled. We also write $\mathscr{A}[\mathscr{P}, l$, INCR $, I,+\infty]$ to denote the property obtained from $\mathscr{A}[\mathscr{P}, l$, INCR, $I, B]$ by replacing $[0, B-1]^{J}$ by $\mathbb{N}^{J}$ in $\left(\mathrm{P}^{\prime}\right)$. Observe that a pseudo-run satisfies $\mathscr{A}[\mathscr{P}, 1, \emptyset,[1, n],+\infty]$ iff it weakly satisfies $\mathscr{P}$. The property $\mathscr{A}[\mathscr{P}, l, \mathrm{INCR}, I,+\infty]$ is exactly the condition we need in the proof of Lemma 4.3 below thanks to the property stated below.

Lemma 4.1. If the pseudo-run $\vec{y}_{2 l-2} \stackrel{\pi_{l-1}^{\prime}}{\longrightarrow} \vec{y}_{2 l-1} \stackrel{\pi_{l}}{\rightarrow} \vec{y}_{2 l} \ldots \stackrel{\pi_{K}}{\rightarrow} \vec{y}_{2 K}$ satisfies the approximation property $\mathscr{A}[\mathscr{P}, l, \mathrm{INCR}, I,+\infty]$, then $\left(\pi_{l-1}^{\prime}\left(\pi_{l}\right)^{n_{l}} \pi_{l}^{\prime}\left(\pi_{l+1}\right)^{n_{l+1}} \cdots\left(\pi_{K}\right)^{n_{K}}, \vec{y}_{2 l-2}\right)$ also satisfies it, for $n_{l}, \ldots, n_{K} \geq 1$. A similar statement does not hold for pseudo-runs satisfying $\mathscr{A}$ (values for components in $J$ might become out of $[0, B-1]$ ) and for runs satisfying $\mathscr{P}$ (component values might become negative). Property $\mathscr{A}[\mathscr{P}, l$, INCR, $I, B]$ can be viewed as a collection of local path increasing formulae in the sense of [2]. 


\subsection{Bounding the length of pseudo-runs}

Let us briefly recall the structure of Rackoff's proof to show that the boundedness problem for VAS is in EXPSPACE. A witness run for unboundedness is of the form $\rho=\vec{x}_{0} \stackrel{*}{\rightarrow} \vec{y} \stackrel{+}{\rightarrow} \vec{y}^{\prime}$ with $\vec{y} \prec \vec{y}^{\prime}$. In [21], it is shown that $\rho$ can be of length at most doubly exponential. In order to get the EXPSPACE upper bound, Savitch's theorem is used. Rackoff's proof to establish the small run property goes as follows. First, a technical lemma shows that if there is some $i$ - $B$-bounded pseudo-run (instance of the approximation property $\mathscr{A}$ ), then there is one of length at most $B^{|\mathscr{T}|^{C}}$ for some constant $C$. The proof essentially shows that existence of such a pseudo-run amounts to solving an inequation system and by using [4], small solutions exist, whence the obtention of a short $i$ - $B$-bounded pseudo-run. The idea of using small solutions of inequation system to solve problems on counter systems dates back from [21, 9] and nowadays, this is a standard proof technique. This proof can be extended to numerous properties on pseudo-runs for which intermediate counter value differences can be expressed in Presburger arithmetic as done in [29, 2]. Then, a proof by induction on the dimension is performed by using this very technical lemma and the ability to repeat sequences of transitions; the proof can be extended when the first intermediate configuration is less or equal to the last configuration of the sequence (leading to the concept of increasing path formula in [2]). This condition allows to perform the induction on the dimension with a unique increasing formula. Unfortunately, generalized unboundedness properties are not increasing in the sense of [2] and therefore Rackoff's proof requires to be extended (but the main ingredients remain). The generalization of the technical lemma is presented below; it is not surprising since generalized unboundedness properties are Presburger-definable properties. However, not only we need to refine the expression $B^{|\mathscr{T}|^{C}}$ in terms of various parameters (length of $\mathscr{P}, \operatorname{scale}(\mathscr{P}), n, \operatorname{scale}(\mathscr{T})$ ) in order to get the final ExPSPACE upper bound (or the PSPACE upper bound with fixed dimension), but also we have to check that the new ingredients in the definition of $\mathscr{A}$ do not prevent us from extending [21, Lemma 4.4]. Finally, it is important to specify the length of small pseudo-runs with respect to parameters from $\mathscr{P}$.

Lemma 4.2. Let $\mathscr{T}$ be a VAS of dimension $n \geq 2, \mathscr{P}$ be a generalized unboundedness property of length $K, l \in[1, K], B \geq 2, I, \mathrm{INCR} \subseteq[1, n]$ and $\rho$ be a pseudo-run satisfying $\mathscr{A}[\mathscr{P}, l, \mathrm{INCR}, I, B]$. Then, there exists a pseudo-run starting by the same pseudo-configuration, satisfying $\mathscr{A}[\mathscr{P}, l$, INCR, $I, B]$ and of length at most $(1+K) \times(\operatorname{scale}(\mathscr{T}) \times \operatorname{scale}(\mathscr{P}) \times B)^{n^{\mathrm{C}_{1}}}$ for some constant $\mathrm{C}_{1}$ independent of $K$, scale $(\mathscr{P})$, $\operatorname{scale}(\mathscr{T}), B$ and $n$.

The length expression in Lemma 4.2 can be certainly refined in terms of card(INCR), $\operatorname{card}(I)$ and $l$ but these values are anyhow bounded by $n$ and $K$ respectively, which is used in Lemma 4.2. For $i \in[0, n]$,

$$
g(i) \stackrel{\text { def }}{=} \begin{cases}(2 \mu)^{n^{\mathrm{c}_{1}}} \text { with } \mu=(1+K) \times \operatorname{scale}(\mathscr{T}) \times \operatorname{scale}(\mathscr{P}) & \text { if } i=0, \\ (2 \mu(\operatorname{maxneg}(\mathscr{T}) \times g(i-1)))^{n^{\mathrm{c}_{1}}}+g(i-1) & \text { if } i>0 .\end{cases}
$$

Lemma 4.3 below is an extension of [21, Lemmas $4.6 \& 4.7]$, see also [2, Lemma 7].

Lemma 4.3. Let $I, \mathrm{INCR} \subseteq[1, n], l \in[1, K]$ and $\rho$ be a pseudo-run satisfying $\mathscr{A}[\mathscr{P}, l, \mathrm{INCR}, I,+\infty]$. Then, there exists a pseudo-run $\rho^{\prime}$ starting from the same pseudo-configuration, satisfying the property $\mathscr{A}[\mathscr{P}, l, \mathrm{INCR}, I,+\infty]$ and of length at most $g(\operatorname{card}(I))$.

In the induction step, we need to take advantage simultaneously of the pigeonhole principle, the induction hypothesis and Lemma 4.2

Proof. Let $\rho=\vec{x}_{2 l-2} \stackrel{\pi_{l-1}^{\prime}}{\rightarrow} \vec{x}_{2 l-1} \stackrel{\pi_{l}}{\rightarrow} \vec{x}_{2 l} \ldots \stackrel{\pi_{K-1}^{\prime}}{\rightarrow} \vec{x}_{2 K-1} \stackrel{\pi_{K}}{\rightarrow} \vec{x}_{2 K}$ be a pseudo-run satisfying the property $\mathscr{A}[\mathscr{P}, l, \mathrm{INCR}, I,+\infty]$. We suppose that $\rho$ is induced by the path $t_{1} \ldots t_{k}$ with $\rho=\vec{u}_{0} \cdots \vec{u}_{k}$ and $f$ : $[2 l-2,2 K] \rightarrow[0, k]$ is the map such that $\vec{x}_{i}=\vec{u}_{f(i)}(f(2 l-2)=0, f(2 K)=k)$. 
The proof is by induction on $i=\operatorname{card}(I)$. If $i=0$, then we apply Lemma 4.2 with $B=2$ and we obtain a pseudo-run satisfying $\mathscr{A}[\mathscr{P}, l, \mathrm{INCR}, I,+\infty]$ leading to the bound $(\mu \times 2)^{n^{\mathrm{l}_{1}}}$.

Now suppose $\operatorname{card}(I)=i+1$ and $J=(I \backslash \operatorname{INCR})$. We pose $B=\operatorname{maxneg}(\mathscr{T}) \times g(i)$. We recall that $\mathscr{T}$ is the current VAS with $n \geq 2$. We perform a case analysis depending where in $\rho$ a value from a component in $J$ is strictly greater than $B-1$ (if any).

Case 1: Every configuration $\vec{z}$ in $\rho$ satisfies $\vec{z}(J) \in[0, B-1]^{J}$, i.e., $\rho$ satisfies $\mathscr{A}[\mathscr{P}, l$, INCR $, I, B]$. Obviously, the case $J=\emptyset$ is captured here. By Lemma 4.2, there is a pseudo-run $\rho^{\prime}$ starting at $\vec{x}_{2 l-2}$ satisfying $\mathscr{A}[\mathscr{P}, l$, INCR $, I, B]$ of length at most $(1+K) \times(\operatorname{scale}(\mathscr{T}) \times \operatorname{scale}(\mathscr{P}) \times B)^{n^{\mathrm{C}_{1}}}$, which is bounded by $(\mu \times(\operatorname{maxneg}(\mathscr{T}) \times g(i)))^{n^{\mathrm{C}_{1}}}$.

Case 2: A value for some component in $J$ is strictly greater than $B-1$ for the first time within the path $\pi_{D}^{\prime}$ for some $D \in[l-1, K-1]$. Let $\alpha$ be the minimal position such that $\vec{u}_{\alpha+1}(J) \notin[0, B-1]^{J}$ and $\alpha+1 \in[f(2 D)+1, f(2 D+1)]$, say $\vec{u}_{\alpha+1}\left(i_{0}\right) \geq B$ for some $i_{0} \in J$. The pseudo-run $\rho$ can be decomposed as follows with $\pi_{D}^{\prime}=\pi_{D}^{1} t_{\alpha+1} \pi_{D}^{2}$ (INCR' is defined few lines below):

$$
\underbrace{\vec{x}_{2 l-2} \stackrel{\pi_{l-1}^{\prime}}{\rightarrow} \vec{x}_{2 l-1} \cdots \vec{x}_{2 D}}_{\rho_{1}}=\underbrace{\vec{x}_{2 D} \stackrel{\pi_{D}^{1}}{\rightarrow} \vec{u}_{\alpha}}_{\rho_{2}} \stackrel{t_{\alpha+1}}{\longrightarrow} \overbrace{\underbrace{\vec{u}_{\alpha+1} \stackrel{\pi_{D}^{2}}{\rightarrow} \vec{x}_{2 D+1} \cdots \vec{x}_{2 K-1} \frac{\pi_{K}}{\rightarrow} \vec{x}_{2 K}}_{\rho_{3}}}^{\text {satisfies } \mathscr{A}\left[\mathscr{P}, D+1, \mathrm{INCR}{ }^{\prime},\left(I \backslash\left\{i_{0}\right\}\right),+\infty\right]}
$$

We shall construct a pseudo-run of the form $\rho_{1}^{\prime} \rho_{2}^{\prime} \rho_{3}^{\prime}$ such that each $\rho_{j}^{\prime}$ is obtained by shortening $\rho_{j}$ and the length of $\rho_{1}^{\prime}$ [resp. $\rho_{2}^{\prime}, \rho_{3}^{\prime}$ ] is bounded by $(\mu \times B)^{n^{c_{1}}}+1$ [resp. $B^{i+1}+1, g(i)+1$ ].

- If $D>l-1$, then we introduce $\mathscr{P}^{\star}=\left(\mathscr{I}_{l}^{\prime}, \ldots, \mathscr{I}_{D}^{\prime}\right)$ with for $l^{\prime \prime} \in[l, D]$ and $j \in[1, n]$, if $\vec{x}_{2 l^{\prime \prime}}(j)-$ $\vec{x}_{2 l^{\prime \prime}-1}(j)>0$ then $\mathscr{I}_{l^{\prime \prime}}^{\prime}(j)=\mathscr{I}_{l^{\prime \prime}}(j) \cap\left[1,+\infty\left[\right.\right.$, otherwise $\mathscr{I}_{l^{\prime \prime}}^{\prime}(j)=\mathscr{I}_{l^{\prime \prime}}(j)$. The construction of $\mathscr{P}^{\star}$ allows us to preserve the set of components in $[l, D]$ whose values can be arbitrarily increased. By Lemma 4.2 , there is a pseudo-run $\rho_{1}^{\prime}=\left(t_{1}^{1} \cdots t_{\beta_{1}}^{1}, \vec{x}_{2 l-2}\right)$ satisfying $\mathscr{A}\left[\mathscr{P}^{\star}, 1\right.$, INCR, I, B] such that $\beta_{1} \leq(\mu \times B)^{n^{\mathrm{c}_{1}}}$. Indeed, scale $(\mathscr{P} \star) \leq \operatorname{scale}(\mathscr{P})$ and the length of $\mathscr{P}^{\star}$ is obviously bounded by $K$. Say $\rho_{1}^{\prime}=\vec{y}_{2 l-2} \stackrel{*}{\rightarrow} \vec{y}_{2 l-1} \stackrel{*}{\rightarrow} \vec{y}_{2 l} \cdots \stackrel{*}{\rightarrow} \vec{y}_{2 D-1} \stackrel{*}{\rightarrow} \vec{y}_{2 D}$. Suppose that $\rho_{1}^{\prime}=\vec{u}_{0}^{1} \cdots \vec{u}_{\beta_{1}}^{1}$ and $f_{1}$ : $[2 l-2,2 D] \rightarrow\left[0, \beta_{1}\right]$ is the map such that $\vec{y}_{i}=\vec{u}_{f_{1}(i)}^{1}\left(f_{1}(2 l-2)=0, f_{1}(2 D)=\beta_{1}\right)$. If $D=l-1$, then $\rho_{1}=\left(t_{1} \cdots t_{\alpha}, \vec{x}_{2 l-2}\right)$ with an analogous decomposition in terms of $\vec{y}_{i}$ 's. We have $\left\{j: \vec{y}_{2 l^{\prime}-1}(j)<\right.$ $\left.\left.\vec{y}_{2 l^{\prime}}(j), l^{\prime} \in[l, D]\right\}=\left\{j: \vec{x}_{2 l^{\prime}-1}(j)<\vec{x}_{2 l^{\prime}}(j), l^{\prime} \in[l, D]\right\} \stackrel{\text { def }}{=} Z\right)$-partly by construction of $\mathscr{P}^{\star}$.

- Now, by the piegonhole principle, there is a pseudo-run $\rho_{2}^{\prime}=\left(t_{1}^{2} \cdots t_{\beta_{2}}^{2}, \vec{y}_{2 D}\right)$ such that $\vec{u}_{\alpha}^{\prime}=\vec{y}_{2 D}+$ $t_{1}^{2}+\cdots+t_{\beta_{2}}^{2}, \vec{u}_{\alpha}^{\prime}(J)=\vec{u}_{\alpha}(J)$ and $\beta_{2}<B^{\operatorname{card}(J)} \leq B^{i+1}$. We pose $\vec{u}_{\alpha+1}^{\prime}=\vec{u}_{\alpha}^{\prime}+t_{\alpha+1}$.

- Finally, observe that $\left(t_{\alpha+2} \cdots t_{k}, \vec{u}_{\alpha+1}^{\prime}\right)$ satisfies $\mathscr{A}\left[\mathscr{P}, D+1, \mathrm{INCR}^{\prime},\left(I \backslash\left\{i_{0}\right\}\right),+\infty\right]$ with $\mathrm{INCR}^{\prime} \stackrel{\text { def }}{=}$ $\mathrm{INCR} \cup Z$. By the induction hypothesis, there is a pseudo-run $\rho_{3}^{\prime}=\left(t_{1}^{3} \cdots t_{\beta_{3}}^{3}, \vec{u}_{\alpha+1}^{\prime}\right)$ satisfying $\mathscr{A}\left[\mathscr{P}, D+1, \mathrm{INCR}^{\prime},\left(I \backslash\left\{i_{0}\right\}\right),+\infty\right]$ and such that $\beta_{3} \leq g(i)$. Because $\vec{u}_{\alpha+1}^{\prime}\left(i_{0}\right) \geq \operatorname{maxneg}(\mathscr{T}) \times$ $g(i), \rho_{3}^{\prime}$ also satisfies $\mathscr{A}\left[\mathscr{P}, D+1, \mathrm{INCR}^{\prime}, I,+\infty\right]$.

Glueing the previous transitions, the pseudo-run $\left(t_{1}^{1} \cdots t_{\beta_{1}}^{1} t_{1}^{2} \cdots t_{\beta_{2}}^{2} t_{\alpha+1} t_{1}^{3} \cdots t_{\beta_{3}}^{3}, \vec{x}_{2 l-2}\right)$ satisfies the approximation property $\mathscr{A}[\mathscr{P}, l, \mathrm{INCR}, I,+\infty]$. and its length is bounded by $(\mu \times B)^{n^{\mathrm{C}_{1}}}+B^{i+1}+g(i)$.

Case 3: A value for some component in $J$ is strictly greater than $B-1$ for the first time within the path $\pi_{D}$ for some $D \in[l, K]$.

The pseudo-run $\rho$ can be written as follows with $\pi_{D}=\pi_{D}^{1} \pi_{D}^{2}$ and $\pi_{D}^{1} \neq \varepsilon$

$$
\vec{x}_{2 l-2} \stackrel{\pi_{l-1}^{\prime}}{\longrightarrow} \vec{x}_{2 l-1} \cdots \vec{x}_{2 D-1} \stackrel{\pi_{D}^{1}}{\rightarrow} \vec{u}_{\alpha+1} \stackrel{\pi_{D}^{2}}{\rightarrow} \vec{x}_{2 D} \cdots \vec{x}_{2 K-1} \stackrel{\pi_{K}}{\rightarrow} \vec{x}_{2 K}
$$


By Lemma 4.1 , the pseudo-run $\rho^{\prime}\left(\pi_{l-1}^{\prime} \pi_{l} \cdots \pi_{D-1}^{\prime}\left(\pi_{D}\right)^{2} \pi_{D}^{\prime} \cdots \pi_{K}, \vec{x}_{2 l-2}\right)$ also satisfies the approximation property $\mathscr{A}[\mathscr{P}, l, \mathrm{INCR}, I,+\infty]$ and can be written as $\vec{x}_{2 l-2} \stackrel{\pi_{l-1}^{\prime}}{\longrightarrow} \vec{x}_{2 l-1} \cdots \vec{x}_{2 D-2} \stackrel{\pi_{D-1}^{\prime} \pi_{D}}{\longrightarrow} \vec{x}_{2 D}=\vec{z}_{2 D-1} \stackrel{\pi_{D}}{\longrightarrow}$ $\vec{z}_{2 D} \stackrel{\pi_{D+1}^{\prime}}{\longrightarrow} \cdots \vec{z}_{2 K-1} \stackrel{\pi_{K}}{\longrightarrow} \vec{z}_{2 K}$. We are therefore back to Case 2 .

Now, we are seeking to bound $g(n)$.

Lemma 4.4. If $\rho$ is a pseudo-run weakly satisfying $\mathscr{P}$, then there is a $\rho^{\prime}$ starting from the same pseudoconfiguration, weakly satisfying $\mathscr{P}$ and of length at most $(\mu \times 2 \times \operatorname{maxneg}(\mathscr{T}))^{n^{(2 n+1) c}}$ for some C $>1$ with $\mu=(1+K) \times \operatorname{scale}(\mathscr{T}) \times \operatorname{scale}(\mathscr{P})$.

Proof. Let us bound $g(n)$. By Lemma 4.3 , for some constant $\mathrm{C}_{2}>\mathrm{C}_{1}$ (for instance $\mathrm{C}_{2}=\mathrm{C}_{1}+1$ ), we have

$$
g(i) \leq \begin{cases}(2 \mu)^{n^{c_{2}}} & \text { if } i=0, \\ (2 \mu(\operatorname{maxneg}(\mathscr{T}) \times g(i-1)))^{n^{c_{2}}} & \text { if } i>0 .\end{cases}
$$

By induction on $i$, we can show that $g(i) \leq\left(v^{i+1}\right)^{n^{(2 i+1) c_{2}}}$ with $v=2 \mu \times \operatorname{maxneg}(\mathscr{T})$. For $i=0$ this is obvious . Otherwise

$$
\begin{aligned}
g(i+1) & \leq(2 \mu \times \operatorname{maxneg}(\mathscr{T}) \times g(i))^{n^{\mathrm{C}_{2}}} \leq\left(v\left(v^{i+1}\right)^{n^{(2 i+1) \mathrm{C}_{2}}}\right)^{n^{\mathrm{C}_{2}}} \leq \ldots \\
& \leq\left(\left(v^{i+2}\right)^{n^{(2 i+1) \mathrm{c}_{2}}}\right)^{n^{\mathrm{C}_{2}}} \leq\left(v^{i+2}\right)^{n^{(2 i+2) \mathrm{C}_{2}}}<\left(v^{i+2}\right)^{n^{(2 i+3) \mathrm{C}_{2}}}
\end{aligned}
$$

Hence, $g(n) \leq\left(v^{n+1}\right)^{n^{(2 n+1) \mathrm{C}_{2}}}$. As soon as $n \geq 2$, there is a constant C s.t. $g(n) \leq(2 \mu \times \operatorname{maxneg}(\mathscr{T}))^{n^{(2 n+1) \mathrm{c}}}$.

Let us conclude the section by the main result of the paper.

Theorem 4.5. (I) The generalized unboundedness problem for VASS is EXPSPACE-complete. (II) For each $n \geq 1$, the generalized unboundedness problem restricted to VASS of dimension at most $n$ is in PSPACE.

\section{Other Applications}

In this section, we draw conclusions from Theorem 4.5. First, as a by-product of Theorem 4.5 and using the reductions from Section 3.3, we can easily regain the exponential-space bound mentioned below.

Corollary 5.1. The regularity detection problem and the strong promptness detection problem are in EXPSPACE. The simultaneous unboundedness problem is EXPSPACE-complete. For each fixed $n \geq 1$, their restriction to VASS of dimension at most $n$ are in PSPACE.

The complexity upper bound for regularity detection problem has been left open in [2]. Decidability of the strong promptness detection problem is established in [27]. The EXPSPACE upper bound has been already stated in [29, 2]. We cannot rely on [29] because of the flaw in [29, Lemma 7.7]. Condition 4. in [2, page 13] does not characterize strong promptness (but only promptness) as shown in Section 3.3. Finally, increasing path formulae from [2] cannot characterize strong promptness detection unlike generalized unboundedness properties. Therefore, we also believe that the upper bound for strong promptness detection is new. Below, we state how the previous results allow us to characterize the computational complexity of reversal-boundedness detection problem for VASS and its variant with weak reversal-boundedness. 
Theorem 5.2. (I) Reversal-boundedness detection problem for VASS is EXPSPACE-complete. (II) For each fixed $n \geq 1$, its restriction to VASS of dimension at most $n$ is in PSPACE. (III) Properties (I) and (II) also hold true for weak reversal-boundedness detection problem.

By Theorem 5.2(I), once an initialized VASS is shown to be reversal-bounded, one can compute effectively semilinear sets corresponding to reachability sets, see recent developments in [26]. The size of the representation of such sets is at least polynomial in the maximal number of reversals. However, we know that an initialized VASS can be bounded but still the cardinality of its reachability set may be nonprimitive recursive, see e.g. [28]. A similar phenomenon occurs with reversal-boundedness, as briefly explained below. In case of reversal-boundedness, the maximal reversal can be nonprimitive recursive in the size of the initialized VASS in the worst-case. Indeed, given $n \geq 0$, one can compute in time polynomial in $n$ an initialized VASS $\left(\mathscr{V}_{n},\left(q_{0}, \vec{x}_{n}\right)\right)$ that generates a finite reachability set of cardinal $\mathscr{O}(A(n))$ for some nonprimitive recursive map $A(\cdot)$ similar to Ackermann function, see e.g., the construction in [14]. Moreover, $\left(\mathscr{V}_{n},\left(q_{0}, \vec{x}_{n}\right)\right)$ can be shown to admit only finite runs, see details in [14]. It is then easy to compute a variant VASS $\mathscr{V}_{n}^{\prime}$ by adding a component and such that each transition of $\mathscr{V}_{n}$ is replaced by itself followed by incrementating the new component and then decrementing it (creating a reversal). Still $\mathscr{V}_{n}^{\prime}$ has no infinite computation, $\left(\mathscr{V}_{n}^{\prime},\left(q_{0}, \vec{x}_{n}^{\prime}\right)\right)$ is reversal-bounded $\left(\vec{x}_{n}^{\prime}\right.$ restricted to the components of $\mathscr{V}_{n}$ is equal to $\left.\vec{x}_{n}\right)$ and its maximal reversal is in $\mathscr{O}(A(n))$.

\section{Concluding Remarks}

We have proved the EXPSPACE-easiness of the generalized unboundedness problem (both the initialized VASS and the generalized unboundedness property are part of the inputs). For example, this allows us to provide the optimal complexity upper bound for the reversal-boundedness detection problems, place boundedness problem, strong promptness detection problem and regularity detection problem. Even though our proof technique is clearly tailored along the lines of [21], we had to provide a series of adaptations in order to get the final EXPSPACE upper bound (and the PSPACE upper bound for fixed dimension). In particular, we advocate the use of witness pseudo-run characterizations (instead of using runs) when there exist decision procedures using coverability graphs.

Let us conclude by possible continuations. Our EXPSPACE proof can be obviously extended by replacing intervals in properties by more complex sets of integers or by adding new constraints between intermediate configurations. The robustness of our proof technique still deserves to be determined. A challenging question is to determine the complexity of checking when a reachability set obtained by an initialized VASS is semilinear. Besides, various subclasses of VASS exist for which decision problems are of lower complexity. For instance, in [20], the boundedness problem is shown to be in PSPACE for a class of VASS with so-called bounded benefit depth. It is unclear for which subclasses of VASS, the generalized unboundedness problem can be solved in polynomial space too.

Acknowledgments: I would like to thank Thomas Wahl (U. of Oxford) and anonymous referees for their suggestions and remarks about a preliminary version of this work.

\section{References}

[1] P. Abdulla and B. Jonsson. Verifying programs with unreliable channels. $I \& C, 127(2): 91-101,1996$.

[2] M. F. Atig and P. Habermehl. On Yen's path logic for Petri nets. In RP'09, volume 5797 of $L N C S$, pages 51-63. Springer, 2009. 
[3] B. Baker and R. Book. Reversal-bounded multipushdown machines. JCSS, 8:315-332, 1974.

[4] I. Borosh and L. Treybig. Bounds on positive integral solutions of linear diophantine equations. AMS, 55:299-304, 1976.

[5] Z. Dang, O. Ibarra, and P. San Pietro. Liveness verification of reversal-bounded multicounter machines with a free counter. In FST\&TCS'01, volume 2245 of $L N C S$, pages 132-143. Springer, 2001.

[6] S. Demri and A. Sangnier. When model checking freeze LTL over counter machines becomes decidable. In FOSSACS'10, volume 6014 of LNCS, pages 176-190. Springer, 2010.

[7] J. Esparza. Decidability and complexity of Petri net problems - an introduction. In Advances in Petri Nets 1998, volume 1491 of LNCS, pages 374-428. Springer, Berlin, 1998.

[8] A. Finkel and A. Sangnier. Reversal-bounded counter machines revisited. In MFCS'08, volume 5162 of LNCS, pages 323-334. Springer, 2008.

[9] E. Gurari and O. Ibarra. An NP-complete number-theoretic problem. In STOC'78, pages 205-215. ACM, 1978.

[10] P. Habermehl. On the complexity of the linear-time mu-calculus for Petri nets. In ICATPN'97, volume 1248 of LNCS, pages 102-116. Springer, 1997.

[11] J. Hopcroft and J. Pansiot. On the reachability problem for 5-dimensional vector addition systems. TCS, 8:135-159, 1979.

[12] R. Howell and L. Rosier. An analysis of the nonemptiness problem for classes of reversal-bounded multicounter machines. JCSS, 34(1):55-74, 1987.

[13] O. Ibarra. Reversal-bounded multicounter machines and their decision problems. JACM, 25(1):116-133, 1978.

[14] M. Jantzen. Complexity of Place/Transition Nets. In Advances in Petri Nets 1986, volume 254 of LNCS, pages 413-434. Springer, 1987.

[15] R. M. Karp and R. E. Miller. Parallel program schemata. JCSS, 3(2):147-195, 1969.

[16] R. Kosaraju. Decidability of reachability in vector addition systems. In STOC'82, pages 267-281, 1982.

[17] R. J. Lipton. The reachability problem requires exponential space. Technical Report 62, Department of Computer Science, Yale University, 1976.

[18] E. Mayr. An algorithm for the general Petri net reachability problem. SIAM Journal of Computing, 13(3):441460, 1984.

[19] M. Minsky. Computation, Finite and Infinite Machines. Prentice Hall, 1967.

[20] M. Praveen and K. Lodaya. Modelchecking counting properties of 1-safe nets with buffers in paraPSPACE. In FST\&TCS'09, pages 347-358. LZI, 2009.

[21] C. Rackoff. The covering and boundedness problems for vector addition systems. TCS, 6(2):223-231, 1978.

[22] W. Reisig and G. Rozenberg, editors. Lectures on Petri Nets I: Basic Models, volume 1491 of LNCS. Springer, 1998.

[23] C. Reutenauer. The Mathematics of Petri nets. Masson and Prentice, 1990.

[24] L. Rosier and H.-C. Yen. A multiparameter analysis of the boundedness problem for vector addition systems. JCSS, 32:105-135, 1986.

[25] A. Sangnier. Vérification de systèmes avec compteurs et pointeurs. Thèse de doctorat, LSV, ENS Cachan, France, 2008.

[26] A. To. Parikh images of regular languages: Complexity and applications. arXiv:1002.1464, January 2010.

[27] R. Valk and M. Jantzen. The residue of vector sets with applications to decidability problems in Petri nets. Acta Informatica, 21:643-674, 1985.

[28] R. Valk and G. Vidal-Naquet. Petri nets and regular languages. JCSS, 23:299-325, 1981.

[29] H.-C. Yen. A unified approach for deciding the existence of certain net paths. $I \& C, 96: 119-137,1992$. 